\title{
Performance Comparison and Evaluation of the Routing Protocols for MANETs Using NS3
}

\author{
Yefa Mai ${ }^{1}$, Yuxia Bai ${ }^{1}$ and Nan Wang ${ }^{2}$ \\ 1. Electrical and Computer Engineering, California State University, Fresno CA 93740, USA \\ 2. Electrical and Computer Engineering, California State University, Fresno CA 93740, USA
}

\begin{abstract}
MANETs (mobile ad hoc networks) contain wireless mobile nodes to construct dynamic networks without the support of fixed infrastructure. Its nodes and hosts are all movable and self-configurable while simultaneously serving as routers to deliver data from sources to their respective destinations. Because routers in MANETs have to cooperate efficiently to meet the performance requirements, routing algorithms play a crucial role in MANET architecture design. In the past, tremendous works have been done on comparison and evaluation of routing algorithms using NS2 (network simulator 2). This paper presents performance evaluations and analyses on the Proactive and Reactive routing protocols using NS3 (network simulator 3), a relatively newer and more advanced version of NS2. This paper will also show simulation results of evaluating the drop rate, throughput, power consumption, and end-to-end delay of MANETs. Lastly, a performance justification of said simulation results will be presented.
\end{abstract}

Key words: MANET, proactive routing, reactive routing, NS3.

\section{Introduction}

MANETs (mobile ad hoc networks) have emerged as a promising solution and research topic since the mid-1990s for its efficient wireless communication between movable nodes where fixed infrastructure is unavailable. Due to the mobility of the nodes, the interconnections between nodes are constantly and dynamically reconfigured. All the nodes in an MANET can move individually in any direction at any time, subsequently updating their interconnections accordingly. The biggest challenge in MANET design is ensuring each node maintains connections with its neighbors while simultaneously directing information traffic to the appropriate destinations.

An MANET is generally used in places where a fixed infrastructure can't be formed due to certain reasons, such as disaster areas, war zones, and emergency sites. All MANET hosts are mobile and can be installed in cars, soldiers, ships, buses, airplanes,

Corresponding author: Nan Wang, Ph.D., associate professor, research fields: wireless communications, cyber physical systems, embedded systems and VLSI design. and emergency response teams, to form temporary networks as shown in Fig. 1. Any of the hosts can move out of range at any time, resulting in a reconfiguration of the network. There are several MANET types, including VANETs (vehicular ad hoc networks), SPANs (smart phone ad hoc networks), iMANETs (Internet based mobile ad hoc networks), and military or tactical MANETs [1].

MANET nodes serve as routers for delivering data from sources to their respective destinations. Routing protocol plays a very important role in MANET design due to the specific characteristics required by this network.

Some research on the topic of MANET Routing Protocol Design has been carried out using NS2 in last several years. For instance, the routing algorithms for AODV (Ad hoc on-demand distance vector), FSR (fisheye state routing), DSDV (destination sequence distance vector), and DSR (dynamic source routing) have already been simulated and compared [2-7]. Study and evaluation of the MANET routing protocols based on different network configurations have been presented [8-11]. 


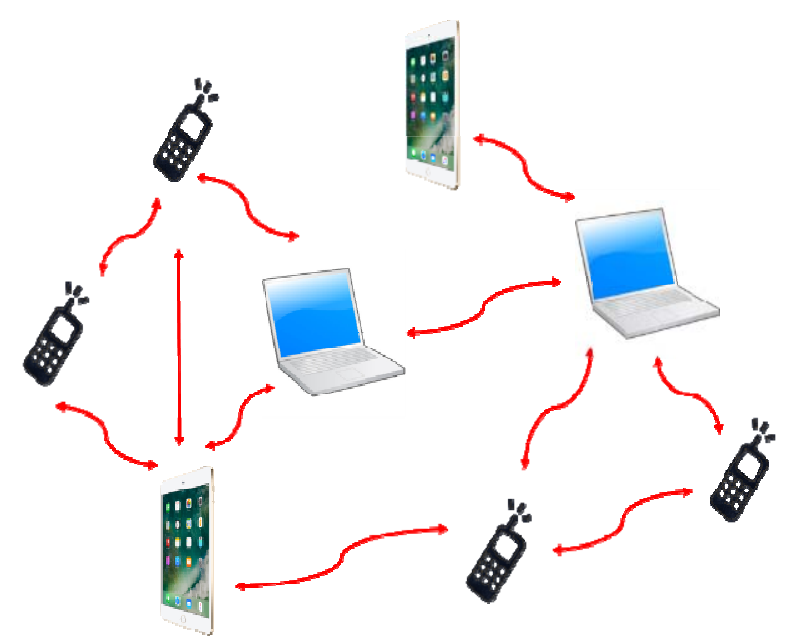

Fig. 1 An MANET (mobile ad-hoc network).

A great deal of work has been done to compare and evaluate the performance of the MANET routing algorithms using NS2 (network simulator 2), which has some limitations compared to NS3 (network simulator 3), a newer and more advanced version of NS2. Detailed simulation modeling methodology of NS3 has rarely been done. In this paper, we examine the performance of several previously aforementioned typical MANET routing protocols using NS3. Information of simulation modeling methodology of NS3 will be presented as well. Among the routing protocols, the DSDV and OLSR algorithms are proactive protocols, and the AODV algorithms are reactive protocols. The simulation results will be compared and justified based on numbers of total nodes and packet size.

The paper is organized as follows: Section 2 discusses five typical MANET routing protocols. Section 3 presents NS3 modeling methodology and simulation set up. The performance justification and analysis are presented in Section 4. Finally, Section 5 concludes the paper.

\section{Ad Hoc Networking Protocols}

In MANET, there are two types of flat routing protocols: proactive routing (table-driven routing protocols) and reactive routing (on-demand routing protocols) to direct data from a host to the destination as shown in Fig. 2.

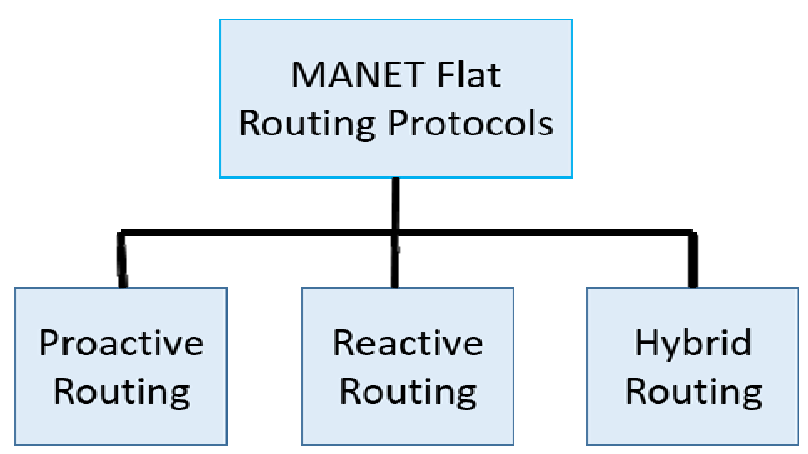

Fig. 2 MANET routing protocols.

\subsection{Proactive Protocols}

In proactive routing protocols, each node maintains and updates one or more tables that contain routing information to other nodes. The information in these tables is consistently updated so as to keep routing information consistent and updated as the network status changes.

The DSDV routing algorithm is based on the classic Bellman-Ford Routing Algorithm. In DSDV, all nodes keep information of neighboring nodes and direct data to subsequent nodes. Before nodes in any route pass a data package to the next nodes, an agreement has to be acknowledged by both. Therefore, all nodes will update routing tables to keep position information in the network consistent and up to date. This causes no disturbance in the route [12].

The OLSR (optimized link state routing protocol) is an IP routing protocol for MANETs, which is based on the link state algorithm. It is one of the proactive protocols, which periodically exchanges a "hello" message to discover and maintain the network. OSLR uses multipoint relaying technique to reduce the flood messages in the networks. Control messages are constructed at each of the nodes to compute next hop destinations for the networks. In OLSR, all the nodes have to be awake, because it uses power and network resources to transmit data via possibly used routes [13].

\subsection{Reactive Protocols}

In reactive protocols (on-demand routing protocols), there is no need for the nodes in the network to keep routing information. Whenever a node needs to send 
data to a destination, a route generation mechanism will create a route based on the current network situation. The route will be canceled when the transaction ends.

In the AODV routing algorithm, nodes work separately and do not keep track of adjacent nodes. Instead, all nodes have information of predefined routes through which data can be delivered to the destination. A route will be formed only when a data arrives at a node to deliver said data to the destination node.

The DSR protocol is a source routed on-demand routing protocol in which all nodes have a route cache to keep routing information from the source nodes. If said source nodes need to send data to a destination, it will first check the route cache for a valid source to destination route. Only then will the source nodes send the packets. If there is no valid route, the source nodes will start to discover and to attempt building routes by sending a route request packet that contains the address of the source and the destination. A route will be only created if the request packet reaches a node that already has a source to node route established.

\subsection{Hybrid Routing Protocols}

An HRP (hybrid routing protocol) uses features of both proactive and reactive protocols. A ZRP (zone routing protocol) is an example of HRPs in which performance is improved by selecting either proactive or reactive protocols based on which is the most efficient algorithm under the current network status.

\section{Simulation Modeling Methodologies}

Simulations have been carried out on NS2 to compare and analyze routing algorithms, such as the DSDV, OLSR, AODV, and DSR, based on various performance metrics. However, performance comparison and analysis between the two classical MANET routing protocol types, proactive and reactive, have rarely been done using NS3 in the Linux Ubuntu operating system. The procedures and simulation results presented in this paper will help MANET researchers and designers tune their systems to meet particular requirements in a more efficient way.

\subsection{Comparison of NS2 and NS3}

A brief comparison between NS2 and NS3 is shown in Table 1 [14].

As shown in Table 1, NS3 is more powerful, flexible and versatile compared to NS2. However, many users are not familiar with NS3 modeling and set up.

\subsection{NS3 Simulation Modeling Methodology}

To establish NS3 simulations, several classes such as core-module.h and network-module.h need to be included. These classes plus their detailed descriptions can be found in NS3 API. Moreover, NS3 employs $\mathrm{C}++$ and Python languages, and several simulation steps need to be followed to start any NS3 simulations. The NS3 simulation procedures are shown in Fig. 3.

Table 1 NS2 versus NS3.

\begin{tabular}{ll}
\hline NS2 & NS3 \\
\hline Not actively maintained and supported & Actively maintained and supported \\
Use TCL as scripting language and is inflexible & Use C++ and Python, is flexible \\
Recompilation is long and easily failed & Recompilation is fast using single command \\
Use nam animator & Use PyViz python visualizer and NetAnim animation \\
Simulation only & Simulation/emulation and DCE environment is provided \\
Hard to get Power Consumption & Power consumption can be achieved \\
No goodput calculation & Goodput is available \\
MAC protocol is fixed & MAC protocol can be defined \\
MANET simulation only & MANET and NOC simulation \\
\hline
\end{tabular}




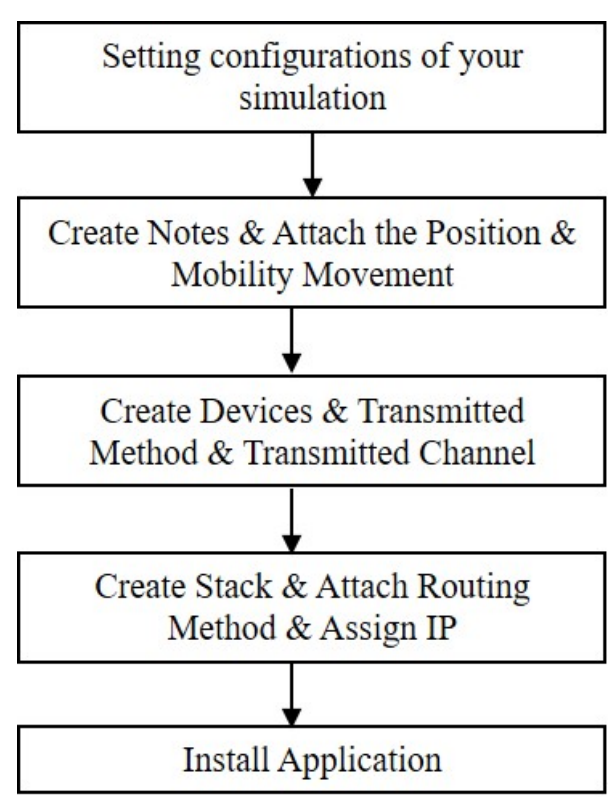

Fig. 3 NS3 simulation procedures.

Table 2 Simulation parameters.

\begin{tabular}{ll}
\hline Parameter & Value \\
\hline Operating System Ubuntu & 14.04 \\
Channel Type & Wireless Channel \\
Number of Nodes & 50 \\
Number of Sink Nodes & $10,30,50$ \\
Node Min Movement Speed (m/s) & 4 \\
Node Max Movement Speed (m/s) & 10 \\
Data Type & UDP \\
Simulation Total Time (s) & 30 \\
MAC Protocol & 802.11 \\
Wi-Fi Transmit Frequency & 2.4 GHz \\
Data Packet Size (bytes) & $64,256,512$ \\
Area of Simulation (m) & $500 * 500$ \\
Radio Prop. Model & Two Ray Ground \\
Routing Protocols & DSDV,OLSR, AODV, DSR \\
Initial Nodes Power (J) & 50 \\
Each Received Consumption & 0.0174 \\
Power (J) &
\end{tabular}

First, all parameter values are presented. Simulation notes are then created and the grid of simulations is defined using the parameters described in the first step. All functions relative to the nodes will be defined as well. The Internet Layer of the devices will be created, which will declare how the data transmitted between the devices and which channel is using the devices. At the same time, the devices are installed according to the nodes. In the transport layer, the IP address, ports and the routing method are set up. The stack for the Internet to store the IP address, ports, and kind of information are also defined. In the application layer, the source nodes and the sink nodes are assigned for the data transition. Lastly, the simulations are ready to carry out with the schedule time. The simulation environment is set up as shown in Table 2.

The four MANET routing protocols were simulated based on the following performance metrics.

- THPT (throughput): ratio of amount of received packets to the duration of simulation time.

- GPT (goodput): total successfully routed data (bytes) to the duration of simulation time.

- PDR (packet delivery ratio): the ratio of the number of packets successfully delivered to the destinations.

- AETED (average end-to-end delay): the average time to deliver a packet from source to the destination.

- PCMP (power consumption): the power consumption due to sink notes receiving packets.

\section{Simulation Results and Justifications}

Through the simulations, we evaluate and analyze the performance upon different simulation setups, such as varied number of nodes (10, 30 and 50), varied packet sizes (64, 256 and 512), etc.

\subsection{Simulation Results}

NS3 simulation results of the MANET with 10 nodes are shown in Table 3 and Fig. 4 respectively.

NS3 simulation results of the MANET with 30 nodes are shown in Table 4 and Fig. 5 respectively.

NS3 simulation results of the MANET with 50 nodes are shown in Table 5 and Fig. 6 respectively.

\subsection{Simulation Result Justification}

Figs. 4-6 present graphical representations of the data seen in Tables 3-5 respectively. From the simulation results, we can draw the following conclusions. 
Table 3 Simulation results (number of node: 10 ).

\begin{tabular}{lllll}
\hline Packets & MTRS & Routing algorithms & & AODV \\
\hline & & DSDV & OLSR & $71,878.8$ \\
\hline \multirow{2}{*}{64} & THPT & $66,666.3$ & $62,381.4$ & $49,939.7$ \\
& GPT & $33,766.6$ & $43,322.0$ & 75.19 \\
& PDR & 78.82 & 80.53 & $3,105.68$ \\
\hline & AETED & $2,781.27$ & $3,560.41$ & 9.24598 \\
\hline & PCMP & 7.82799 & 11.0894 & $71,354.0$ \\
\multirow{2}{*}{256} & THPT & $76,716.4$ & $57,963.3$ & $64,188.2$ \\
& GPT & $69,014.5$ & $52,141.7$ & 94.71 \\
& PDR & 98.422 & 97.49 & $3,882.94$ \\
\hline & AETED & $2,797.74$ & $4,720.51$ & 12.7253 \\
\hline & PCMP & 12.1615 & 14.2795 & $84,518.7$ \\
& THPT & $80,007.8$ & $60,781.1$ & $79,949.6$ \\
& GPT & $75,724.3$ & $57,516.6$ & 82.24 \\
& PDR & 97.39 & 92.67 & $8,403.1$ \\
\hline
\end{tabular}

(a) Throughput

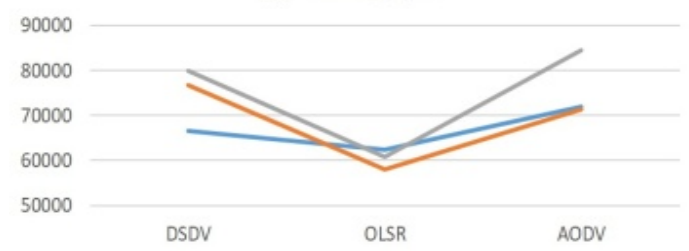

(c) PDR

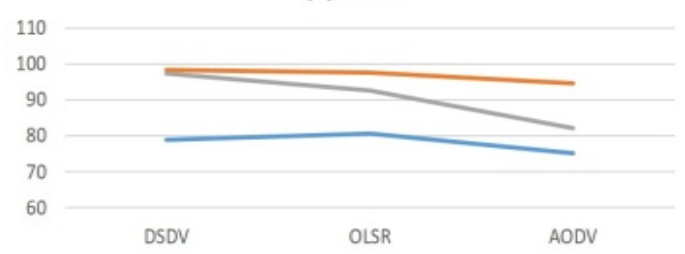

(e) PCMP

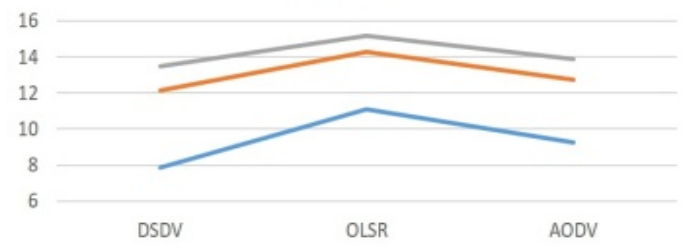

Fig. 4 NS3 simulation results with 10 nodes. (b) Goodput

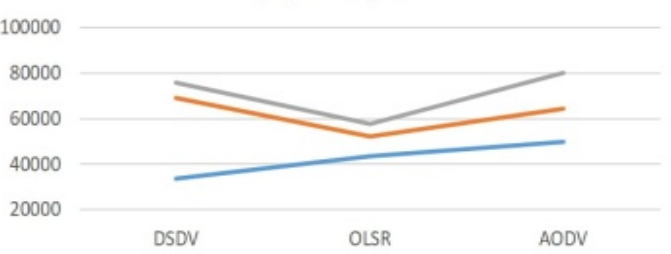

(d) AETED (ns)

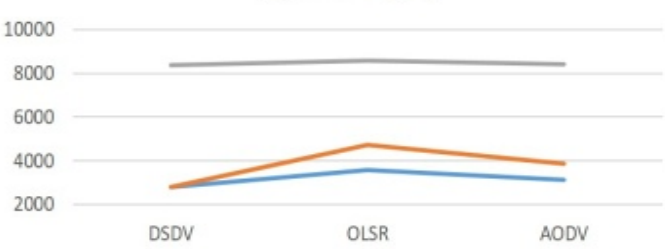

Packet Size

-64
-256 
Table 4 Simulation results (number of node: 30 ).

\begin{tabular}{lllll}
\hline Packets & MTRS & Routing algorithms & & AODV \\
\hline & & DSDV & OLSR & $66,536.7$ \\
\multirow{2}{*}{64} & THPT & $65,433.7$ & $52,124.7$ & $46,118.9$ \\
& GPT & $45,373.3$ & $36,166.8$ & 73.04 \\
& PDR & 74.47 & 82.0 & $4,030.56$ \\
\hline & AETED & $2,894.79$ & $4,101.25$ & 8.79967 \\
\hline & PCMP & 7.9509 & 10.025 & $70,099.1$ \\
& THPT & $70,415.6$ & $64,057.5$ & $62,880.2$ \\
& GPT & $63,213.4$ & $57,593.2$ & 84.86 \\
& PDR & 91.48 & 99.77 & $6,363.95$ \\
\hline & AETED & $5,953.08$ & $5,220.23$ & 12.2665 \\
\hline & PCMP & 12.1005 & 12.7167 & $74,372.9$ \\
& THPT & $75,829.4$ & $64,854.5$ & $70,113.5$ \\
& GPT & $71,642.3$ & $61,330.1$ & 43.56 \\
& PDR & 89.76 & 98.99 & $10,806.7$ \\
\hline
\end{tabular}

(a) Throughput

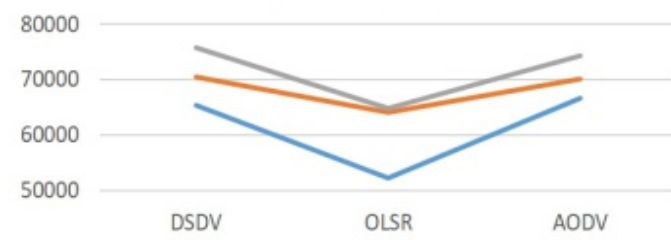

(c) PDR

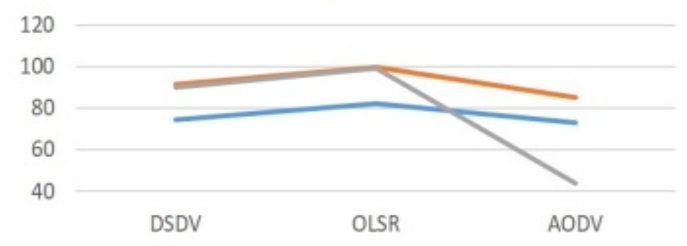

(e) PCMP

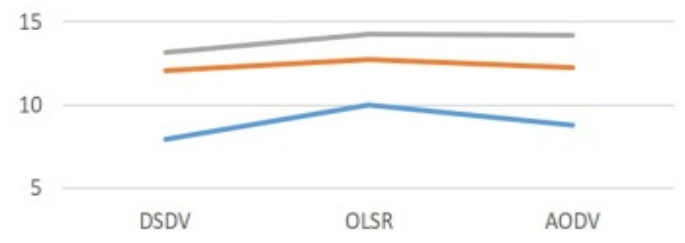

(b) Goodput

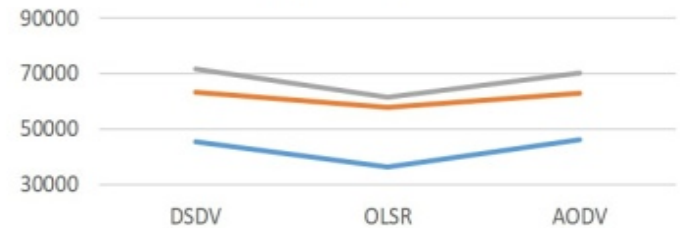

(d) AETED (ns)

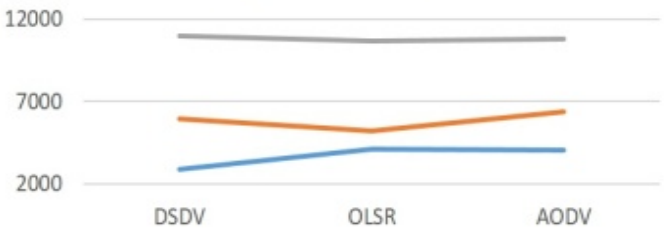

Packet Size

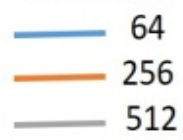

Fig. 5 NS3 simulation results with 30 nodes. 
Table 5 Simulation results (number of node: 50 ).

\begin{tabular}{cllll}
\hline Packets & MTRS & Routing algorithms & \\
\hline \multirow{3}{*}{64} & & DSDV & OLSR & AODV \\
\hline & THPT & $61,849.1$ & $52,867.7$ & $71,192.9$ \\
& GPT & $42,880.2$ & $36,646.8$ & $49,456.2$ \\
& PDR & 75.03 & 73.28 & 76.67 \\
& AETED & $3,140.23$ & $4,304.24$ & $4,202.31$ \\
\hline & PCMP & 7.96209 & 9.25128 & 7.76671 \\
\hline \multirow{2}{*}{256} & THPT & $66,878.9$ & $57,749.6$ & $76,346.9$ \\
& GPT & $59,943.6$ & $51,841.0$ & $68,315.0$ \\
& PDR & 94.82 & 99.95 & 92.59 \\
& AETED & $7,399.38$ & $6,131.61$ & $6,601.19$ \\
\hline & PCMP & 12.0916 & 12.5217 & 11.0943 \\
\hline & THPT & $65,851.2$ & $74,551.5$ & $77,599.0$ \\
& GPT & $62,030.0$ & $70,542.6$ & $73,166.3$ \\
& PDR & 94.89 & 97.7491 & 94.6062 \\
& AETED & $12,343.8$ & $12,131.8$ & $11,744.2$ \\
\hline
\end{tabular}

(a) Throughput

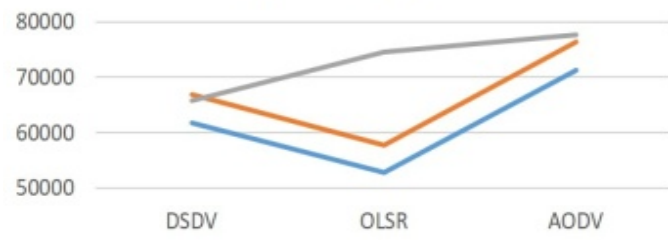

(c) PDR

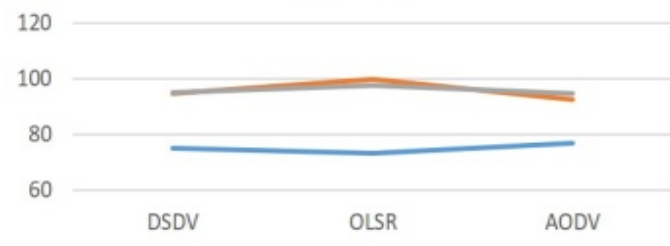

(e) PCMP

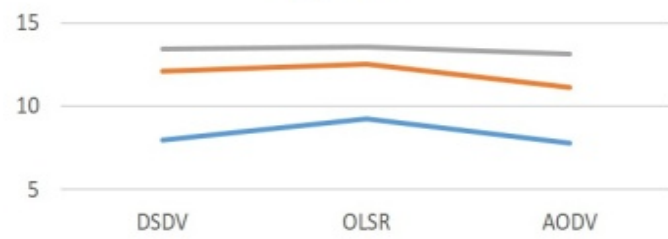

OLSR

AODV (b) Goodput

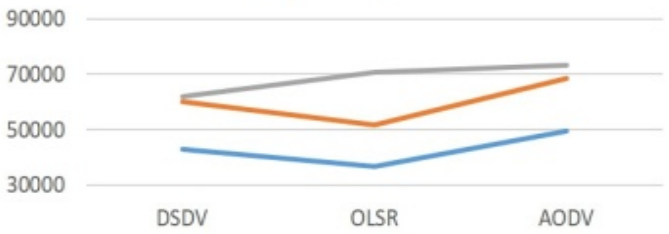

(d) AETED (ns)

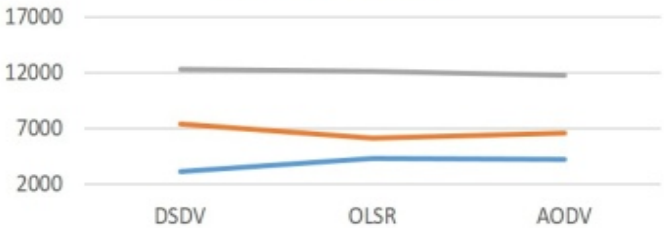

Packet Size

-64
-256
-512

Fig. 6 NS3 simulation results with 50 nodes. 
As the packet size increased, throughput, goodput, packet delivery ratio, average end-to-end delay and power consumption are increased for all routing protocols on the MANETs with 10, 30 and 50 nodes.

The throughput and goodput for the AODV of the reactive type becomes better or more competitive as the number of nodes increases. That of the other three routing protocols worsens. The AODV of the reactive type performs the best.

According to packet delivery ratio, as the number of nodes and packet size increase, the OLSR of the proactive type becomes the best performer, while the performances of the other two algorithms remain at a similar level.

When comparing the throughput between the routing protocols, the DSDV of proactive types and the AODV of reactive types are found to be very competitive. AODV performs the best when the number of nodes and packet sizes are increased. The OLSR of proactive types is the worst performing protocol.

Base on power consumption, the OLSR algorithm performs the worst, while the other two protocols are very competitive, with the DSDV slightly over performs the AODV in this category.

When comparing the proactive protocol type with the reactive protocol type, it is easy to conclude that the performance of the two major routing protocols (DSDV and AODV) is competitive when the network size is relatively small (10 nodes). As the size of the network increases (30 and 50 nodes), the reactive routing protocols (AODV) become dominant in all performance categories. On the other hand, the packet size plays a non-crucial role in the comparison.

\section{Conclusion}

Several MANET routing protocols have been simulated and evaluated in this paper. NS3 simulation modeling methodologies have been presented. Test results demonstrated that the AODV algorithm of the reactive type performs better in terms of throughput and average end-to-end delay, while the OLSR of the reactive type is a little better among the routing algorithms in terms of packet delivery ratio. As the size of the network increases, the reactive protocols of the AODV routing algorithm become dominant in all performance categories, while the influence of the packet size is insignificant. The results of the work will help the researchers and designers better understand the performance of the major routing protocols in an MANET, so as to tune their MANET simulation systems based on various requirements. We hope to show the performance of the routing protocols in real world MANET applications in the future.

\section{References}

[1] Mobile Ad Hoc Network, WIKIPEDIA, https://en.wikipedia.org/wiki/Mobile_ad_hoc_network.

[2] Alslaim, M. N., Alaqel, H. A., and Zaghloul, S. S. 2014. "A Comparative Study of MANET Routing Protocols." Third International Conference on E-Technologies and Networks for Development (ICeND), 178-82.

[3] Ullah, F., Amin, M., and Ghaffar, H. 2010. "Simulating AODV and DSDV for a Dynamic Wireless Sensor Networks.” IJCSNS International Journal of Computer Science and Network Security 10 (7).

[4] Kaushik1, S. S., and Deshmukh, P. R. 2009. "Comparison of Effectiveness of AODV, DSDV and DSR Routing Protocols in Mobile Ad-Hoc Network.” International Journal of Information Technology and Knowledge Management 2 (2).

[5] Udayakumar, P., and Ambhaikar, A. 2009. "Experimenatal Comparison of AODV and DSR Protocols.” International J. of Eng. Research \& Indu. Appls. 2 (III).

[6] Tuteja, A., Gujrl, R., and Thalia, S. 2010. "Comparative Performance Analysis of DSDV, AODV and DSR Routing Protocols in MANET Using NS2.” International Conference on Advances in Computer Engineering.

[7] Rahman, M. A., Anwar, F., Naeem, J., and Abedin, M. S. M. 2010. “A Simulation Based Performance Comparison of Routing Protocol on Mobile Ad-hoc Network (Proactive, Reactive and Hybrid).” International Conference on Computer and Communication Engineering, 11-3.

[8] Bai, Y. X., Mai, Y. F., and Wang, N. 2017. "Performance Comparison and Evaluation of the Proactive and Reactive Routing Protocols for MANETs.” In Proceedings of IEEE WTS Conference. 
[9] Jorg, D. O. 2003. "Performance Comparison of MANET Routing Protocols in Different Network Sizes.” University of Berne, Switzerland.

[10] Corson, S., and Macker, J. 1999. "Mobile Ad Hoc Networking (MANET): Routing Protocol Performance Issues and Evaluation Considerations.” University of Mary Land.

[11] Kr. Sarma, S., Dhar Choudhury, S., Ahmed, A. U., and Hannan, A. 2010. "Efficiency Analysis of Routing Protocols for Mobile Ad Hoc Networks for Heterogeneity." International Journal of Computer Networks 2 (1).
[12] Misra, P. 1999. "Routing Protocols for Ad Hoc Mobile Wireless

Networks." http://www.cse.wustl.edu/ jain/cis788-99/ftp/adhoc_routi $\mathrm{ng} /$.

[13] Jacquet, P., Muhlethaler, P., Clausen, T., Laouiti, A., Qayyum, A., and Viennot, L. 2001. "Optimized Link State Routing Protocol for Ad Hoc Network.” In Proceedings of IEEE International INMIC.

[14] "Difference between NS-2 and NS-3." 2016. https://rishikeshteke.wordpress.com/tag/difference-betwe en-ns2-and-ns3/. 